\title{
OBITUARIO
}

\section{In Memoriam doctora Nelly Carrillo Tarazona de Espinoza \\ (1927- 2017)}

\section{In Memoriam doctora Nelly Carrillo Tarazona de Espinoza \\ (1927 - 2017)}

\section{Javier Icochea Monteza}

Email Javier Icochea: anfirep@yahoo.es

Biólogo, investigador independiente

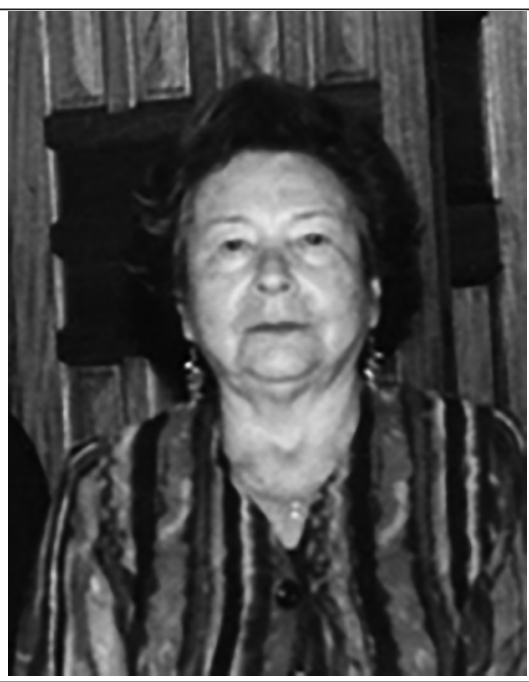

La doctora Nelly Carrillo Tarazona de Espinoza, falleció el 12 de agosto del 2017. Nació en Chiquián, provincia de Bolognesi en el departamento de Ancash el 2 de noviembre de 1927. Estudió ciencias biológicas en la Escuela Instituto de Ciencias Biológicas, Facultad de Ciencias de la Universidad Nacional Mayor de San Marcos (UNMSM), egresando en el año 1950, año a partir del cual la UNMSM comenzó a otorgar el título de Biólogo. Sustento su tesis doctoral en la UNMSM en 1973, titulada "Una nueva especie del género Sibynomorphus (Serpentes: Colubridae)".

La doctora Carrillo dedicó sus investigaciones al estudio de anfibios y reptiles, enfatizando en los reptiles, como lo atestiguan sus publicaciones. Ella sucedió a Jehan Vellard, médico de nacionalidad francesa que contribuyó al desarrollo y ampliación de los estudios herpetológicos y del Laboratorio de Herpetología del Museo de Historia Natural de la Universidad Nacional Mayor de San Marcos. Posteriormente, la doctora Carrillo continuaría con el desarrollo de esta linea de investigación desempeñándose como Jefa del Departamento de Herpetología durante más de 25 años, consagrando sus esfuerzos al cuidado y mantenimiento de la importante colección científica de dicho departamento.

\section{Citación:}

Icochea J. 2017. In Memoriam doctora Nelly Carrillo Tarazona de Espinoza (1927-2017). Revista peruana de biología 24(3): 229 - 230 (octubre 2017). doi: http://dx.doi.org/10.15381/rpb.v24i3.13912 
Como profesional y persona, fue generosa tanto en brindar sus conocimientos como en su trato. Ella demostraba destreza en la manipulación de las serpientes y otros reptiles, lo cual realizaba con firmeza, seguridad y precaución. Estas destrezas y sus actividades llamaban la atención porque en esos años aun no era frecuente ver mujeres en esta especialidad y demostrando su gran habilidad.

Tuve la oportunidad de trabajar con ella alrededor de 12 ańos, desarrollando conjuntamente en los últimos tres años, el inventario de los reptiles del Perú, publicando la lista preliminar que ha sido bastante usada por los especialistas e interesados en nuestra diversidad biológica.

En homenaje a la Dra. Nelly Carrillo han sido descritas y nominadas dos especies:

$>$ Telmatobius carrillae Morales, 1988: Es una rana andina descrita por el herpetólogo Peruano Víctor Morales.

$>$ Euspondylus nellycarrillae Köhler \& Lehr, 2004: Es una lagartija andina descrita por los herpetólogos alemanes Gunther Köhler y Edgar Lehr.

Sin duda, seguirá siendo extrañada por quienes la conocimos y trabajamos con ella. Descanse en paz estimada Doctora.

La presente nota fue posible a la información proporcionada por Rosario Espinoza (hija de la Dra. Nelly Carrillo), las Dras. Bertha Herrera Alarcón de Loja y Norma Chirichigno Fonseca (compañeras de estudios de la Dra. Nelly Carrillo) y ccon la colaboración de la Dra. Magda Chanco, Mg. Martha Valdivia Cuya, y el Dr. César Aguilar Puntriano, actual Jefe del departamento de Herpetología del MUSM, a quienes les quedo muy agradecido.

\section{Publicaciones científicas de la Dra. Nelly Carrillo de Espinoza}

[1966] Carrillo, N. Contribución al conocimiento de los Boideos peruanos (Boidae, Ophidia, Reptilia). Revista de Ciencias, (535-538): 86-136.

[1966] Carrillo, N. Acción del veneno de Hapalopus limensis. Mem. Inst. Butantan, Simp. Internac., 33(3): 799-808.
[1966] Carrillo, N. Contribución al conocimiento de los Boideos peruanos (Boidae, Ophidia, Reptilia). Public. Mus. Hist. Nat. (UNMSM), Serie A, Zoología, 21: 1-51.

[1970] Carrillo, N. Contribución al conocimiento de los Reptiles del Perú (Squamata, Crocodylia, Testudinata: Reptilia). Public. Mus. Hist. Nat. (UNMSM), Serie A, Zoología, 22: 1-64.

[1974] Carrillo, N. Una nueva especie del género Sibynomorphus (Serpentes: Colubridae). Public. Mus. Hist. Nat. (UNMSM), Serie A, Zoología, 24: 1-11.

[1977] Carrillo, N. Arańas y serpientes muy venenosas en el Departamento de Lima. Public. Mus. Hist. Nat. (UNMSM), Serie Divulgación, 8: 1-8.

[1978] Carrillo, N. Serpientes venenosas del Perú: $1^{\circ}$ Parte. Boletín de la Colonia Suiza en el Perú. Setiembre: 47-48.

[1978] Carrillo, N. Serpientes venenosas del Perú: $2^{\circ}$ Parte. Boletín de la Colonia Suiza en el Perú. Noviembre: 47-48.

[1978] Mittermeir, R.; A. G. Rhodin, F. Medem, P. Soini, M. S. Hoogmoed \& N. Carrillo. Distribution of the Soutamerican Chelid turtle Phrynops gibbus with observations on habitat and reproduction. Herpetologica 34: 94-100.

[1983] Carrillo, N. Contribución al conocimiento de las serpientes venenosas del Perú de las familias Viperidae, Elapidae, e Hydrophiidae (Ophidia:Reptilia). Public. Mus. Hist. Nat. (UNMSM), Serie A, Zoología, 30: 1-55.

[1983] Carrillo, N. List of Peruvian Anolis with distributional data (Sauria: Iguanidae). Pp: 406-411. In: A.G. Rhodin \& K. Miyata, (Eds.), Advances in Herpetology and Evolutionary Biology. Cambridge, Massachusetts.

[1985] Carrillo, N. \& G. Lamas. Un nuevo registro de Tortuga Terrestre para el Perú (Reptilia: Testudinata). Public. Mus Hist. Nat. (UNMSM), Serie A, Zoología, 31: 1-7.

[1987] Carrillo, N. Hallazgo de Eretmochelys imbricata bissa (Ruppel) en la costa norte del Perú (Testudinata:Cheloniidae). Nota inicial. Biota 94: 40-45.

[1990] Morales, V. R., N. Carrillo \& H. Ortega. El material Tipo de Peces, Anfibios y Reptiles en el Museo de Historia Natural de la Universidad Nacional Mayor de San Marcos. Public. Mus Hist. Nat. (UNMSM), Serie A, Zoología, 33: 1-7.

[1990] Carrillo, N. Nombres populares de los reptiles del Perú. Boletín de Lima, 70: 23-28.

[1990] Carrillo, N.; Rothenstein, D., Salas, A. \& Werner, Y. Radiation and Convergence among desert geckos: Phyllodactylus species resembling both Ptyodactylus and Stenodactylus. AmphibiaReptilia, 11: 1-13.

[1995] Carrillo, N. \& J. Icochea. Lista Taxonómica Preliminar de los Reptiles Vivientes del Perú. Public. Mus Hist. Nat. (UNMSM) Serie A, Zoología, 49: 1-27.

[2004] Lehr, E., N. Carrillo \& P. Hocking. A new species of Drymoluber (Reptilia: Squamata: Colubridae) from southeastern Peru. Copeia 2004(1): 46-52. 